\title{
Resampling Marked Point Processes
}

DIMITRIS N. POLITIS University of California, San Diego,

La Jolla, California

EFSTATHIOS PAPARODITIS University of Cyprus, Nicosia, Cyprus

JOSEPH P. ROMANO Stanford University, Stanford,

California

\section{INTRODUCTION AND NOTATION}

Suppose $\left\{X(t), t \in \mathbb{R}^{d}\right\}$ is a homogeneous random field in d dimensions, with $d \in \mathrm{Z}^{+}$, that is, a collection of real-valued random variables $X(t)$ that are indexed by the continuous parameter $t \in \mathbb{R}^{d}$. In the important special case where $d=1$, the random fiedd $\{X(t)\}$ is just a continuous timc, stationary stochastic process. The probability law of the random field $\left(X(t), t \in R^{\prime \prime}\right)$ will be denoted by $P_{X}$. We will generally assume that $E X(t)^{2}<\infty$, in which case homogeneity (i.e., strict stationarity) implies weak stationarity, namacly that for any $\mathrm{t}, \mathrm{h} \in \mathrm{R}^{d}$, $E X(\mathrm{t})=\mu$, and $\operatorname{Con}(X(\mathrm{t}), X(\mathrm{t}+\mathrm{h}))=R(\mathrm{~h})$; in ollser words, $l X X(t)$ and $\operatorname{Cou}\left(X^{\prime}(t), X^{\prime}(t+h)\right)$ do not depend on $t$ at illt.

Our objective is statistical inference pertaining to feitures of the unknown probability law $P_{X}$ on the basis of data; in particular. this paper will focus on estimation of the common mean $/ 2$. For the casc where the data are of the form $\{X(t), t \in E\}$, with $\mathrm{E}$ being a finite subset of the rectangular lattice $Z^{d}$, different block-resampling iecliniques have been developed in the literature; see, for examplc. Hall (1985), 
Carlstein (1986), Künsch (1989), Lahiri (1991), Liu and Singh (1992), Politis and Romano (1992a,b,c, 1993, 1994), Raïs (1992), and Sherman and Carlstein $(1994,1996)$. However, in many important cases, for example, queucing theory, spatial statistics, mining and geostatistics, meteorology, etc., the data correspond to observations of $X(t)$ at nonlattice, irregularly spaced points. For instance, if $d=1, X(t)$ might represent the required service time for a customer arriving at a service station at time t. If $d=2$, $X(t)$ might represent a measurement of the quality or quantity of the ore found in location $t$, or a measurement of precipitation at location $t$ during a fixed time interval, etc. As a matter of fact, in case $d>1$, irregularly spaced data seem to be the rule rather than the exception; see, for example, Cressic (1991), Karr (1991), and Ripley (1981).

A useful and parsimonious way to model the irregularly scattered tpoints is to assume they are generated by at homogeneous Poisson point process observable on a compact subset $K \in \mathbb{R}^{d}$, and assumed to be independent of the random field $\{X(t)\}$; see Karr $(1986,1991)$ for a thorough discussion on the plausibility of the Poisson assumption. So let $N$ denote such a homogeneous Poisson process on $\mathbf{R}^{d}$, independent of $\{X(\mathrm{t})\}$, and possessing mean measure $\Lambda$, that is, $E N(A)=\Lambda(A)$ for any set $A \subset \mathbf{R}^{d}$; note that homogeneity of the process allows us to write $\Lambda(A)=\lambda|A|$, where $\lambda$ is a positive constant signifying the "rate" of the process, and $|\cdot|$ denotes Lebesgue measure (volume). The point process $N$ can then be expressed as $N=\sum_{i} \epsilon_{t}$, where $\epsilon_{t}$ is a point mass at t, i.e., $\epsilon_{t}(A)$ is 1 or 0 according to whether $t \in A$ or not; in other words, $N$ is a random (counting) measure on $\mathbf{R}^{d}$. The expected number of t-points to be found in $A$ is $\Lambda(A)$, whereas the actual number of $t$-points found in set $A$ is given by $N(A)$. The joint (product) probability law of the random field $\{X(t)\}$ and the point process $N$ will be denoted by $P$. The observations are then described via the "marked point process" $\tilde{N}=\sum_{i} \epsilon_{[t, ., Y(t,)]}$, which is just the point process $N$ with each t-point being "marked" by the value of $X$ at that point.

Hence, in this paper, our objective will be interval estimation of $\mu$ on the basis of measurements of the value of $X(\cdot)$ at a finite number of generally non lattice, irregularly spaced points $t \in \mathbf{R}^{\prime}$. The observed marked point process is then defined as the collection of pairs $\left\{\left(t_{j}, X\left(t_{j}\right)\right)\right.$. $j=1, \ldots, N(K)\}$, where $\left\{\mathrm{t}_{j}\right\}$ are the points at which the $\left\{X\left(\mathrm{t}_{j}\right)\right\}$ "marks" happen to be observed; see Daley and Vera-Jones (1988), Karr (1991), or Krickeberg (1982) for more details on marked point processes.

The paper is organized as follows: Section 2 contains some useful notions on mixing, and some necessary background on mean estimation, in Section 3 the marked point process "circular" bootstrap is introduced and studied, while in Section 4 the marked point process "block" bootstrap is introduced and studied; some concluding remarks are presented in Section 5, while all proofs are deferred to Section 6.

\section{SOME BACKGROUND AND A USEFUL LEMMA ON MIXING}

The continuous parameter random field $\left\{X(\mathrm{t}), \mathrm{t} \in \mathrm{R}^{d}\right\}$ will be assumed to satisfy a certain weak dependence condition that will be quantified in terms of mixing coefficients. Let $\rho(\cdot, \cdot)$ denote sup-distance (i.e., the distance arising from the $l_{\infty}$ norm) on $\mathbf{R}^{d}$; the strong mixing cocfficients of Rosenblatt (1985) are then defined as

$$
\begin{gathered}
\alpha_{N^{\prime}}(k) \equiv \sup _{E_{1}, E_{2} \subset \mathbb{R}^{\prime}}\left\{P\left(A_{1} \cap A_{2}\right)-P\left(A_{1}\right) P\left(A_{2}\right) \mid: A_{i} \in \mathcal{F}\left(E_{i}\right), i=1,2,\right. \\
\left.\rho\left(E_{1}, E_{2}\right) \geq k\right\}
\end{gathered}
$$

where $\mathcal{F}\left(E_{i}\right)$ is the $\sigma$-algebra generated by $\left\{X(t), t \in E_{i}\right\}$. Alternatively, in it random field set-up where $d>1$, it is now customary to consider mixing coefficients that in general also depend on the size (volume) of the sets considered; sce for example Doukhan (1994). Thus define $\alpha_{X}\left(k ; l_{1}, l_{2}\right) \equiv$

$$
\begin{aligned}
& \sup _{E_{1}, E_{2} \subset R^{d}} \| P\left(A_{1} \cap A_{2}\right)-P\left(A_{1}\right) P\left(A_{2}\right)\left|: A_{i} \in \mathcal{F}\left(E_{i}\right),\right| E_{i} \mid \leq l_{i}, i=1,2 . \\
& \rho\left(E_{1}, E_{2}\right) \geq k \text {. }
\end{aligned}
$$

Note that $\alpha_{X}\left(k ; l_{1}, l_{2}\right) \leq \alpha_{X}(k)$, and that in essence $\alpha_{X} \cdot(k)=\alpha_{X}(k: \infty, \infty)$. $\wedge$ random field is said to be strong mixing if $\lim _{k \rightarrow \infty} \alpha_{x}(k)=0$. There are many interesting examples of strong mixing random fields; sec Rosenblatt (1985). However, there is a big class of random fields of great interest in spatial statistics, namely Gibbs (Markov) random fields in $d>1$ dimensions, that are $n o t$ strong mixing, but instead satisfy a condition on the decay of the $\alpha_{x}\left(k ; l_{1}, l_{2}\right)$ coefficients; sce Doukhan (1994).

Nevertheless, for our results a yet weaker notion of mixing is required. So we define the cocfficients

$$
\begin{aligned}
\bar{\alpha}_{X}(k ; l) & \equiv \sup \| P\left(A_{1} \cap A_{2}\right)-P\left(A_{1}\right) P\left(A_{2}\right) \mid: A_{i} \in \mathcal{F}\left(E_{i}\right), i=1,2 . \\
E_{2} & \left.=E_{1}+\mathrm{t},\left|E_{1}\right|=\left|E_{2}\right| \leq l, \rho\left(E_{1}, E_{2}\right) \geq k\right\}
\end{aligned}
$$

where the supremum is now taken over all compact and convex sets $E_{1} \subset \mathrm{R}^{\prime \prime}$. and over all $\mathrm{t} \in \mathrm{R}^{d}$ such that $\rho\left(E_{1}, E_{1}+\mathrm{t}\right) \geq k$. As before, we may also define $\hat{\alpha}_{X}(k)=\tilde{\alpha}_{X}(k ; \infty)$.

It is easy now to see that $\bar{\alpha}_{X}(k) \leq \alpha_{X}(k)$, so that if the random field is $\alpha$ strong mixing, then it will necessarily be $\bar{\alpha}$-strong mixing as well, implying $\lim _{k \rightarrow \infty} \bar{\alpha}_{X}(k)=0$. In other words, $\bar{\alpha}$-mixing is easier to satisfy than regular 
$\alpha$-mixing. Similarly, $\bar{\alpha}_{X}\left(k ; l_{1}\right) \leq \alpha_{X}\left(k ; l_{1}, l_{1}\right)$, and if the $\alpha_{X}\left(k ; l_{1}, l_{1}\right)$ coefficients are small, then the same will be true for the $\bar{\alpha}_{x}\left(k ; l_{1}\right)$ coefficients; our bootstrap results will consequently be based on this weaker notion. Sec Doukhan (1994), Roussas and Ioannides (1987), and Ivanov and Leonenko (1986, p. 34) for discussion and references on strong mixing coefficients.

We now give a useful lemma, its essence being that mixing properties of the continuous parameter random field are inherited by the observed marked point process; the lemma is a generalization of a result of Masry (1988), who considered renewal point processes on the real line (case $d=1$ ).

LEMMA 1: Let $N_{g}$ be a general Poisson process (not necessarily homogeneous) on $\mathrm{R}^{d}$, possessing mean measure $\Lambda_{g}$, and assumed to be independent of the random field $\left\{X(t), \mathbf{t} \in \mathbf{R}^{d}\right\}$. Let $E_{1}, E_{2}$ be two subsets of $\mathbf{R}^{d}$ such that $\rho\left(E_{1}, E_{2}\right)=k>0$, and define $\bar{Y}_{i}=N_{g}\left(E_{i}\right)^{-1} \int_{E_{i}} X(\mathrm{t}) N(\mathrm{dt})$ and $\bar{Y}_{i}=$ $\left(\Lambda_{g}\left(E_{i}\right)\right)^{-i} \int_{E_{i}} X(\mathrm{t}) N(\mathrm{dt})$ for $i=1,2$; also assume that $E|X(\mathrm{t})|^{p}=C_{p}<\infty$ for some $p>2$. Then

$$
\left|\operatorname{Cov}\left(\bar{Y}_{1}, \hat{Y}_{2}\right)\right| \leq 10 C_{p}^{2 / p}\left(\alpha_{X}\left(k ;\left|E_{1}\right|,\left|E_{2}\right|\right)\right)^{1-2 / p}
$$

and

$$
\left|\operatorname{Cov}\left(\bar{Y}_{1}, \bar{Y}_{2}\right)\right| \leq 10 C_{p}^{2 / p}\left(\alpha_{X}\left(k ;\left|E_{1}\right|,\left|E_{2}\right|\right)\right)^{1-2 / p}
$$

If $E_{1}, E_{2}$ are compact, convex, and are translates of one another, that is, if $E_{1}=E_{2}+\mathrm{t}$, then we also have

$$
\left|\operatorname{Cov}\left(\tilde{Y}_{1}, \bar{Y}_{2}\right)\right| \leq 10 C_{p}^{2 / p}\left(\bar{\alpha}_{X}\left(k ;\left|E_{1}\right|\right)\right)^{1-2 / p}
$$

and

$$
\left|\operatorname{Cov}\left(\bar{Y}_{1}, \bar{Y}_{2}\right)\right| \leq 10 C_{p}^{2 / p}\left(\bar{\alpha}_{X}\left(k ;\left|E_{1}\right|\right)\right)^{1-2 / p}
$$

Although we will subsequently use Lemma 1 in the special case of a homogeneous Poisson process, the lemma's generality is noteworthy.

We now consider estimation of the mean of the random field $\mu=E X(\mathrm{t})$ on the basis of observing $\{X(t)\}$ for the $t$-points generated by the homogeneous Poisson process $N$ over the compact, convex set $K \subset \mathbf{R}^{d}$. It is natural to estimate $\mu$ by the sample mean which-as hinted at in Lemma $1-$ can be defined in two asymptotically equivalent ways:

$$
\tilde{X}_{K} \equiv \frac{1}{\lambda|K|} \int_{K} X(\mathrm{t}) N(\mathrm{dt})
$$

and

$$
\bar{X}_{K} \equiv \frac{1}{N(K)} \int_{K} X(\mathrm{t}) N(\mathrm{~d} \mathrm{t})
$$

the difference between the two being division by expected or actual sample size, respectively. Obviously, if $\lambda$ is unknown, then our only practical choice is $\tilde{X}_{\mathcal{K}}$. Note that Karr (1986) presents some arguments in favor of using $\bar{X}_{\mathcal{K}}$ even if $\lambda$ is known; however, in this paper we will study both $\tilde{X}_{K}$ and $\bar{X}_{K}$ in the interest of completeness.

It is immediate that $\bar{X}_{K}$ is unbiased for $\mu ; \bar{X}_{K}$ is also unbiased as a conditioning (on $N$ ) argument shows. See, for instance, Karr (1986) where it is also shown that under some regularity assumptions, as $|K| \rightarrow \infty, \tilde{X}_{K}$ and $\bar{X}_{K}$ are both consistent and asymptotically normal at rate $\sqrt{|K|}$ with the same asymptotic variance. We explicitly give Karr's (1986) theorem below as it will be useful for our bootstrap theory.

THEOREM 1 [Karr, 1986]: Let $R(\mathrm{t})=\operatorname{Cov}(X(0), X(\mathrm{t}))$, and assume that

$$
\int R(\mathrm{t}) \mathrm{d} \mathrm{t}<\infty
$$

where $\int$ is short-hand for $\int_{\mathrm{R}^{d}}$. Also assume that

$$
\frac{1}{\sqrt{|K|}} \int_{K}(X(\mathrm{t})-\mu) \mathrm{dt} \stackrel{c}{\Rightarrow} N\left(0, \int R(\mathrm{t}) d \mathrm{t}\right)
$$

as diam $(K) \rightarrow \infty$, where diam $(K)$ denotes the supremum of the diameters of all $l_{\infty}$ balls contained in $K$. Then, as diam $(K) \rightarrow \infty$, we have

$$
\sqrt{|K|}\left(\bar{X}_{K}-\mu\right) \stackrel{\mathcal{L}}{\Rightarrow} N\left(0, \sigma^{2}\right), \quad \sqrt{|K|}\left(\bar{X}_{K}-\mu\right) \stackrel{\mathcal{L}}{\Rightarrow} N\left(0, \sigma^{2}\right)
$$

and furthermore

$$
\lim \operatorname{Var}\left(\sqrt{|K|} \bar{X}_{K}\right)=\lim \operatorname{Var}\left(\sqrt{|K|} \bar{X}_{K}\right)=\sigma^{2} \equiv \int R(\mathrm{t}) \mathrm{dt}+\lambda^{-1} R(0)
$$

Note that we have taken the liberty of correcting an obvious typo in the variance formula (3.10) of Karr (1986). To verify the assumptions of Karr's theorem, the following lemma may be used.

LEMMA 2: If $E|X(\mathbf{t})|^{2+\delta}<\infty$ for some $\delta>0$, and $\bar{\alpha}_{X}\left(k ; l_{1}\right) \leq$ const. $\left(1+l_{1}\right)^{\gamma d d^{-1}} k^{-d-\epsilon}$ for some $\epsilon>2 d / \delta$, and some $\gamma<\frac{\delta(c \delta-2 d)}{2 d \delta(1+\delta)}$, then equations (J) and (2) hold true.

Different sufficient conditions for equations (1) and (2) are given in Yadrenko (1983). Nevertheless, to actually use the asymptotic normality 
of the sample mean to construct conlidence intervals for the mean $\mu$, the asymptotic variance must be explicitly estimated. While it is relatively easy to estimate $R(0)$, and $\lambda$ is consistentiy estimable by $N(K) /|K|$. consisten estimation of $\int R(t) d t$ is not a trivial matter, particularly in the calse of the irregularly spaced data considered here.

The resampling methodology that is introduced in this paper is able to yield confidence intervals for the mean without explicit estimation of the asymptotic variance; alternatively, the resampling method may provide an estimate of the asymptotic variance to be used in connection with the asymptotic normality result of Karr (1986). The "circular" resampling methodology of the next section uses a blocking argument similar to the circular bootstrap of Politis and Romano (1992c, 1993), while the "block" resampling methodology of our Section 4 employs arguments similar to the block bootstrap of Künsch (1989) and Liu and Singh (1992).

\section{3. "CIRCULAR" BOOTSTRAP FOR MARKED POINT PROCESSES}

Our goal will be to construct bootstrap confidence intervals for $\mu$ on the basis of observing $\{X(t)\}$ for the t-points generated by the Poisson process $N$ over the compact, convex set $K \subset \mathbf{R}^{d}$. In this section we will further assume that $K$ is a "rectangle," i.e., that $K=\left\{t=\left(t_{1}, \ldots, t_{d}\right)\right.$ : $\left.0 \leq t_{i} \leq K_{i}, i=1, \ldots, d\right)$; in the next section the general case of $K$ being possibly nonrectangular (but convex) will be addressed. The proposed circular bootstrap for marked point processes is described as follows.

1. Begin by imagining that $K$ is "wrapped around" on a compact torus; in other words, we interpret the index $\mathrm{t}$ as being modulo $K$. If $\mathrm{t} \notin K$, we will redefine $\mathrm{t}=\mathrm{t}($ modulo $K)$, where the $i$ th coordinate of vector $\mathrm{t}($ modulo $K)$ is $t_{i}$ (modulo $K_{i}$ ). With this redefinition, we have data $X(t)$ even if $\mathrm{t} \notin K$.

2. Let $c=c(K)$ be a number in $(0,1)$ depending on $K$ in a way that is made precise in Theorem 2, and define a scaled-down replica of $K$ by $B=\{c t: t \in K\}$, where $t=\left(t_{1}, \ldots, t_{d}\right)$ and $c t=\left(c t_{1}, \ldots, c t_{d}\right) ; B$ has the same shape as $K$ but smaller dimensions. Also define the displaced sets $B+y$, and $l e l l=\left[1 / c^{d}\right]$, where $[\cdot]$ denotes the integer part.

3. Gencrate random points $Y_{1}, Y_{2}, \ldots . Y_{1}$ independent and identically distributed from a uniform distribution on $K$, and define

$$
\tilde{X}^{*} \equiv l^{-1} \sum_{i=1}^{l} \frac{1}{\lambda|B|} \int_{B+\mathrm{Y},} X(\mathrm{t}) N(\mathrm{dt})
$$

and

$$
\bar{x}^{*} \equiv r^{-1} \sum_{i=1}^{1} \frac{1}{N\left(B+\mathrm{Y}_{i}\right)} \int_{u+\mathrm{y}_{i}} X(\mathrm{t}) N(\mathrm{dt})
$$

4. This generation of the points $Y_{1}, Y_{2}, \ldots, Y_{1}$ and subsequently of $\bar{x}$. and $X^{*}$ is governed by a probability mechanism which we will denote by $P^{*}$; note that the generation is performed conditionally on the marked point process data that were actually obscrved.

5. Let $P\left(\sqrt{|K|}\left(\bar{X}_{K}-\mu\right) \leq x\right)$ denote the distribution function of the sample mean (centered and normalized), and let $P^{*}\left(\sqrt{|K|}\left(\bar{X}^{*}-E^{*} \bar{X}^{*}\right) \leq x\right)$ and $P^{*}\left(\sqrt{|K|}\left(\bar{X}^{*}-E^{*} \bar{X}^{*}\right) \leq x\right)$ denote the conditional (given the marked point process data) distribution function of its bootstrap counterparts; $E^{*}$ and $V a r^{*}$ denote expected value and variance, respectively, under the probability mechanism $P^{*}$.

An intuitive way of visualizing the construction of $\bar{X}^{*}$ and $\bar{X}^{*}$ is to imagine a "re-tiling" of an area comparable to the rectangle $K$ by putting sideby-side the small rectangles $B+Y_{i}$, carrying along at the same time the $t$ points and their corresponding $X$-marks that the marked point process $\bar{N}$ originally generated in $B+Y_{i}$ as a final step, recalculate the sample mean of the re-tiled process to get $\bar{X}^{*}$ and $\bar{X}^{*}$.

We are now ready to state our main results.

THEOREM 2 Assume equations (I) and (2), let E|X(t)| $\left.\right|^{6+\delta}<\infty$, where $\delta>0$, and assume that $R(0)=\operatorname{Var}(X(t))>0$. Also assume that $\bar{\alpha}_{X}\left(k ; l_{1}\right) \leq$ const. $\left(1+l_{1}\right)^{\bar{\gamma}} k^{-\bar{\beta}}$ for some $\bar{\beta}>3 d$, and $0 \leq \bar{\gamma} \leq \bar{\beta} / d$. Let $\min _{i} K_{i} \rightarrow \infty$. $\max _{i} K_{i}=O\left(\min _{i} K_{i}\right)$, and let $c=c(K) \rightarrow 0$, but in such a way that $c^{d}|K| \rightarrow \infty$. Then

$$
E^{*} \bar{X}^{*}=\tilde{X}_{K}, \quad \frac{\operatorname{Var} \tilde{X}^{*}}{\operatorname{Var} \bar{X}_{K}} \stackrel{p}{\rightarrow} 1
$$

and

$$
\sup _{x}\left|P^{*}\left(\sqrt{|K|}\left(\tilde{X}^{*}-\tilde{X}_{K}\right) \leq x\right)-P\left(\sqrt{|K|}\left(\tilde{X}_{K}-\mu\right) \leq x\right)\right| \stackrel{P}{\longrightarrow} 0
$$

REMARK 1: The result of Theorem 2 could be compactly expressed as

$$
d_{2}\left(P^{*}\left(\sqrt{|K|}\left(\tilde{X}^{*}-\bar{X}_{K}\right) \leq x\right), P\left(\sqrt{|K|}\left(\bar{X}_{K}-\mu\right) \leq x\right)\right) \stackrel{p}{\rightarrow} 0
$$

where $d_{2}(\cdot, \cdot)$ is Mallows metric between distributions-sec Bickel and Freedman (1981) or Shao and Tu (1995, p. 73). Convergence in $d_{2}$ is stron- 
ger than weak convergence, as it also implies convergence of the first two moments. Nevertheless, this compact expression would obscure the fact that we have exact equality of the first moments here, as opposed to only approximate equality as in Theorem 4 of our next section.

REMARK 2: Also recall that in Lemma 2 we gave a sufficient mixing condition that-together with our moment condition-implies equations (1) and (2); however, since in general there exist different sufficient conditions, we followed Karr (1986) in placing equations (1) and (2) in the assumptions of the theorems.

THEOREM 3: Under the conditions of Theorem 2 we have

$$
E^{*} \bar{X}^{*}=\bar{X}_{K}, \quad \frac{\operatorname{Var}^{*} \bar{X}^{*}}{\operatorname{Var}_{\bar{X}}} \stackrel{p}{\rightarrow} 1
$$

and

$$
\sup _{x}\left|P^{*}\left(\sqrt{|K|}\left(\bar{X}^{*}-\bar{X}_{K^{\prime}}\right) \leq x\right)-P\left(\sqrt{|K|}\left(\bar{X}_{K}-\mu\right) \leq x\right)\right| \stackrel{P}{\rightarrow} 0
$$

REMARK 3: Although we have stated the results only for the sample mean (in its two forms), an application of the $\delta$-method (see Bickel and Freedman, 1981) immediately shows that our resampling methodology is also valid for smooth functions of the sample mean. The circular bootstrap (and the block bootstrap discussed in Section 4) can be extended to other mean-like statistical functionals as well, for example, appropriately differentiable statistics (see Künsch, 1989; Liu and Singh, 1992), or general linear statistics (sce Politis and Romano, 1993).

So far we have motivated the introduction of resampling for marked point processes in terms of by-passing the difficult problem of estimating the asymptotic variance $\sigma^{2}$ which is required in order to use the asymptotic normality of the sample mean for confidence intervals. Nevertheless, the bootstrap typically has a further advantage as compared with the asymptotic normal distribution, namely that it yields a more accurate distribution approximation; in other words, the bootstrap is typically "higher order accurate"-sec, e.g., Efron and Tibshirani (1993) or Shao and Tu (1995) and references therein.

Nevertheless, the property of higher order accuracy will apply only to the bootstrap distribution of the standardized or studentized sample mean. For example, the block bootstrap in the case of stationary sequences in discrete time was recently shown to be higher order accurate; sce Lahiri (1991) for the standardized sample mean, and Götze and Künsch (1996) for the stu- dentized sample mean. The same higher order accuracy property characterizes the circular bootstrap; see Politis and Romano (1992c).

Although the necessary tools (for instance, Edgeworth expansions for the distribution of the sample mean) are not yet available to prove higher order accuracy of the bootstrap in a marked point process setting, we conjecture that this higher order accuracy of the standardized or studentized simple mean indeed obtains. With this in mind, we offer the following easy corollary of Theorems 2 and 3, that has to do with the bootstrap distribution of the standardized sample mean. A similar result would be true for the studentized sample mean, in which case a double (or itcrated) bootstrap (cf. Hall, 1992) would be required if we intend to studentize $\bar{X}_{K}$ and $\bar{X}_{K}$ using the bootstrap variance estimator.

COROLLARY 1: Under the conditions of Theorem 2, we also have

$$
\sup _{x}\left|P^{*}\left(\frac{\tilde{X}^{*}-\bar{X}_{K}}{\sqrt{\operatorname{Var} \bar{X}^{*}}} \leq x\right)-P\left(\frac{\tilde{X}_{K^{*}}-\mu}{\sqrt{\operatorname{Var} \tilde{X}_{K^{*}}}} \leq x\right)\right| \stackrel{r}{\rightarrow} 0
$$

and

$$
\sup _{x}\left|P^{*}\left(\frac{\bar{X}^{*}-\bar{X}_{K}}{\sqrt{\operatorname{Var}^{*} \bar{X}^{*}}} \leq x\right)-P\left(\frac{\bar{X}_{K}-\mu}{\sqrt{\operatorname{Var} \bar{X}_{K}}} \leq x\right)\right| \stackrel{P}{\rightarrow} 0
$$

\section{4. "BLOCK" BOOTSTRAP FOR MARKED POINT PROCESSES}

Now our observation region $K$ can be any compact, convex subset of $\mathrm{R}^{d}$; for our asymptotic results, $K$ will be assumed to expand uniformly in all directions, that is, we will assume that $\operatorname{diam}(K) \rightarrow \infty$. As before, $\operatorname{diam}(K)$ is the supremum of the diameters of all $I_{\infty}$ balis contained in $K$, and we also define $\operatorname{Diam}(K)$ to be the infimum of the diameters of all $/_{\infty}$ balls that contain $K$. The block bootstrap for marked point processes is described as follows.

1. As in the previous section, let $c=c(K)$ be a number in $(0,1)$ depending on $K$, and define a scaled-down replica of $K$ by $B=\{c t: t \in K\}$, where $t=\left(t_{1}, \ldots, t_{d}\right)$ and $c t=\left(c t_{1}, \ldots, c t_{d}\right)$. However, since the wraparound will not be used here, we define the set of "allowed" displacements $K_{1-c}=\left\{y \in \mathbf{R}^{\prime l}: B+y \subset K\right\}$, and as before we let $l=\left[1 / c^{d}\right]$. 
2. Generate random points $Y_{1}, Y_{2}, \ldots, Y_{1}$ independent and identically distributed from a uniform distribution on $K_{1-c}$, and let

$$
\bar{X}^{*} \equiv I^{-1} \sum_{i=1}^{t} \frac{1}{\lambda|B|} \int_{B+\gamma_{i}} X(\mathrm{t}) N(\mathrm{dt})
$$

and

$$
\ddot{X}^{*} \equiv I^{-1} \sum_{i=1}^{l} \frac{1}{N\left(B+\mathrm{Y}_{i}\right)} \int_{B+\mathrm{Y}_{i}} X(\mathrm{t}) N(\mathrm{~d} \mathrm{t})
$$

This generation of the points $\mathrm{Y}_{1}, \mathrm{Y}_{2}, \ldots, \mathrm{Y}_{1}$ and subsequently of $\bar{X}^{*}$ and $\bar{X}^{*}$ is governed by a probability mechanism which we will denote by $P^{*}$, with moments denoted by $E^{*}, V a r^{*}$, etc. Note again that this generation is done conditionally on the marked point process data observed; thus $P^{*}$ is really a conditional probability.

3. Let $P^{*}\left(\sqrt{|K|}\left(\bar{X}^{*}-E^{*} \tilde{X}^{*}\right) \leq x\right)$ and $P^{*}\left(\sqrt{|K|}\left(\tilde{X}^{*}-E^{*} \tilde{X}^{*}\right) \leq x\right)$ denote the conditional (given the marked point process data) distribution functions of the bootstrap sample means.

We are now ready to state another set of consistency results.

THEOREM 4: Assume equations (1) and (2), let $E|X(\mathrm{t})|^{6+\delta}<\infty$, where $\delta>0$, and assume that $R(0)=\operatorname{Var}(X(\mathrm{t}))>0$. Also assume that $\bar{\alpha}_{X}\left(k ; l_{1}\right) \leq$ const. $\left(1+l_{1}\right)^{\dot{\gamma}} k^{-\bar{\beta}}$ for some $\bar{\beta}>3 d$, and $0 \leq \bar{\gamma} \leq \bar{\beta} / d$. Let diam $(K) \rightarrow \infty$. $\operatorname{Diam}(K)=O(\operatorname{diam}(K))$, and let $c=c(K) \rightarrow 0$, but in such a way that $c^{d}|K| \rightarrow \infty$. Then

$$
\frac{\operatorname{Var} \tilde{X}^{*}}{\operatorname{Var} \tilde{X}_{K}} \stackrel{p}{\rightarrow} 1
$$

almd

$$
\sup _{x}\left|P^{*}\left(\sqrt{|K|}\left(\tilde{X}^{*}-E^{*} \tilde{X}^{*}\right) \leq x\right)-P\left(\sqrt{|K|}\left(\bar{X}_{K}-\mu\right) \leq x\right)\right| \stackrel{p}{\longrightarrow} 0
$$

THEOREM S: Under the conditions of Theorem 4, we have

$$
\frac{\operatorname{Var}^{*} \bar{X}^{*}}{\operatorname{Var} \bar{X}_{K}} \stackrel{P}{\rightarrow} 1
$$

and

$$
\sup _{x}\left|P^{*}\left(\sqrt{|K|}\left(\bar{X}^{*}-E^{*} \bar{X}^{*}\right) \leq x\right)-P\left(\sqrt{|K|}\left(\bar{X}_{K}-\mu\right) \leq x\right)\right| \stackrel{P}{\longrightarrow} 0
$$

REMARK 4: Note that $E^{*} \bar{X}^{*} \neq \bar{X}_{K}$; instead, $E^{*} \bar{X}^{*}=\bar{X}_{K}+O_{P}(c)$, as the proof of Theorem 4 shows. Therefore, in order not to introduce bias in the bootstrap distribution, it is necessary to center the bootstrap distribution around its bootstrap mean (thus forcing it to have mean zero as in the true distribution of the sample mean); see Lahiri (1991) and Politis and Romano (1992c, 1993) for a similar discussion in the discrete-time casc.

REMARK 5: In comparing the circular bootstrap of Section 3 with the block bootstrap of this section, we note that the block bootstrap is valid even for nonrectangular, convex observation regions $K$, at the expense of having explicitly to center the bootstrap distribution-'see previous Remark - which the circular bootstrap does automatically.

Similarly to Corollary 1 , we now offer a result on the block bootstrap distribution of the standardized sample mean in anticipation of its possible higher order accuracy properties.

COROLLARY 2: Under the conditions of Theorem 4, we also have

$$
\sup _{x}\left|P^{*}\left(\frac{\tilde{X}^{*}-\tilde{X}_{K}}{\sqrt{V^{*} r^{*} \tilde{X}^{*}}} \leq x\right)-P\left(\frac{\bar{X}_{K}-\mu}{\sqrt{\operatorname{Var} \bar{X}_{K}}} \leq x\right)\right| \stackrel{p}{\rightarrow} 0
$$

and

$$
\sup _{x}\left|P^{*}\left(\frac{\bar{X}^{*}-\bar{X}_{K}}{\sqrt{\operatorname{Var} \bar{X}^{*}}} \leq x\right)-P\left(\frac{\bar{X}_{K}-\mu}{\sqrt{\operatorname{Var} \bar{X}_{K}}} \leq x\right)\right| \stackrel{P}{\rightarrow} 0
$$

\section{CONCLUDING REMARKS}

In this paper, we have introduced two different (but closely related) resampling techniques for marked point processes, and have shown that they both fead to consistent estimation of the sampling distribution of the sample mean; thus, the bootstrap estimate of sampling distribution can be used effectively for the construction of confidence intervals and hypothesis testing . regarding the unknown true mean $\mu$.

The proposed techniques may be viewed as variants of the well-known circular and block bootstrap methods originally designed for data observed over points on a rectangular integer lattice. Some further comments regarding the implementation of the methods are given below:

1. For practical implementation, note that since it is possible for us to generate as many pseudo-replicates of $\bar{x}^{*}, \bar{x}^{*}, \tilde{X}^{*}$, and $\bar{x}^{*}$ as we wish. 
the aforementioned circular and block bootstrap distributions $P^{*}$ and $P^{*}$ (as well as their moments $E^{*}, E^{*}, V_{a r}$, and $V_{a r}{ }^{*}$ ) can be evaluated approximately by a Monte Carlo procedure simply by looking at the empirical distribution (and moments) of the corresponding generated pseudo-replicates.

2. It is interesting to note that it is not necessary to take the uniform distribution on $K$ and $K_{1-c}$ als the distribution used to generate the i.i.d. points $Y_{1}, Y_{2}, \ldots, Y_{1}$ that are central to the circular and block bootstrap procedures. For example, the (discrete) uniform distribution on $K \cap(h \mathbf{Z})^{d}$ and $K_{1-c} \cap(h \mathbf{Z})^{d}$ can insteal be used respectively, for the construction of the circular and block bootstrap, for marked point processes without affecting the validity of our asymptotic results; here $h Z=\{h k ; k \in Z\}=\{\ldots,-2 h,-h, 0, h, 2 h, 3 h, \ldots\}$, and $h$ is a positive real number that is either a constant, or in general may depend on $K$ but in such a way that guarantees that the cardinality of the set $K \cap(h Z)^{d}$, and the cardinality of $K_{1-r} \cap(h Z)^{d}$ both tend to infinity as $\operatorname{diam}(K) \rightarrow \infty$.

3. Our bootstrap results are in principle generalizable to the case where the t-points are generated by a Poisson process $N_{k}$, that is not necessarily homogencous; it is for this reason that our Lemma $I$ is stated in such a gencral form. Nevertheless, since in effect the bootstrap for the sample mean will not work unless the sample mean is known to be asymptotically normal, a new version of Karr's theorem would also be required which will not rely on the homogeneity of the Poisson process involved; such a central limit theorem is expected to have rate $\sqrt{\Lambda_{g}(K)}$ as opposed to the $\sqrt{|K|}$ rate of Karr's theorem. Also required would be appropriate restrictions on the mean measure $\Lambda_{g}$ of the general Poisson prossess $N_{g}$; for example, a natural requirement would be to assume that $\Lambda_{g}$ is absolutely continuous with respect to Lebesgue measure on $\mathrm{R}^{t}$, with Radon-Nikodym derivative $\lambda_{x}(t)$ satisfying $\lambda_{g}(t)>\epsilon$ for all $t$, where $\epsilon$ is a positive constant.

4. In general, it is not even necessary to take $l=\left[1 / c^{d}\right]$ for the methods to work. To be more specific, our Corollaries 1 and 2 are stated in a way that makes them remain true verbatim even if $I$ is taken as being different from $\left[1 / c^{d}\right]$, as long as $l \rightarrow \infty$ as $\operatorname{diam}(K) \rightarrow \infty$; in contrast, an explicit renormalization is required for our theorems to remain true if we omit the $l=\left[1 / c^{d}\right]$ (or even $l \sim\left[1 / c^{d}\right]$ ) assumption. Nonetheless, we conjecture that - under some extra conditions - the simple choice $I=\left[1 / c^{d}\right]$ will be required for higher order accuracy results, that is, in order to have the right-hand side of equations (3)-(6) of order $o_{p}(1 /|K|)$.
5. Last but not least is the issue of "optimally" choosing the design parameter $c$ in practice, again with the point of view of improving estimation accuracy. Although our first-order consistency results (for example Corollaries 1 and 2) remain true for any choice of $c=c(K)$ satisfying $c \rightarrow 0$ but $c^{d}\left|K^{\prime}\right| \rightarrow \infty$ as $\operatorname{diam}\left(K^{\prime}\right) \rightarrow \infty$, it is highly plausible that there is an "optimal" choice of $c$ as a function of $K$ that will minimize the right-hand side of equations (3)-(6). Thus, the issues of higher order accuracy and of optimally choosing $c$ are intertwined, and will be the subject of further research. Note, however, that the quest for the optimal $c$ ultimately depends on the optimality criterion employed. For example, improving the accuracy of distribution estimation will generally entail a different optimal choice of $c$ as compared with the one required for improving the accuracy of bootstrap variance estimation; see Hall et al. (1996) for a thorough discussion on the analogous problem of optimal block size in the time series casc.

\section{TECHNICAL PROOFS}

PROOF OF LEMMA 1: Consider first the identity

$$
\begin{aligned}
& E^{N_{x}\left(E_{1}\right) \cdot N_{x}\left(E_{2}\right)} \bar{Y}_{1} \bar{Y}_{2}-E^{N_{x}\left(E_{1}\right) \cdot N_{x}\left(E_{2}\right)} \bar{Y}_{1} E^{N_{x}\left(E_{1}\right), N_{x}\left(E_{2}\right)} \bar{Y}_{2} \\
& =E^{N_{R}\left(E_{1}\right) \cdot N_{R}\left(E_{2}\right)}\left\{E\left(\bar{Y}_{1} \bar{Y}_{2} \mid N_{R}\right)-E\left(\bar{Y}_{1} \mid N_{R}\right) E\left(\bar{Y}_{2} \mid N_{g}\right)\right\}+
\end{aligned}
$$

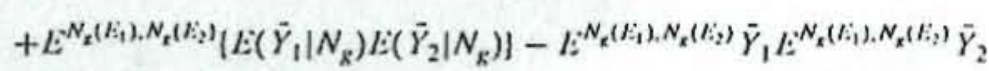

where $E^{N_{R}\left(E_{1}\right) \cdot N_{x}\left(E_{2}\right)} \bar{Y}$ is an alternative short-hand notation for the conditional expectation $E\left(\bar{Y} \mid N_{k}\left(E_{1}\right), N_{k}\left(E_{2}\right)\right)$. Note that $E\left(\bar{Y}_{i} \mid N_{k}\right)$ is at function of just the $\mathrm{t}_{1}^{(i)}, \mathrm{t}_{2}^{(i)}, \ldots, \mathrm{t}_{N_{R}\left(E_{)}\right)}^{(i)}$ points that were generated by the point process $N_{g}$ in the $\operatorname{set} E_{i}$, for $i=1,2$. Also note that $\mathrm{t}_{1}^{(1)}, \mathrm{t}_{2}^{(1)}, \ldots, \mathrm{t}_{N_{1}\left(E_{1}\right)}^{(1)}$ and $\mathrm{t}_{1}^{(2)}, \mathrm{t}_{2}^{(2)}, \ldots, \mathrm{t}_{N_{t}\left(E_{3}\right)}^{(2)}$ are two different collections of t-points, i.e., there are no common t-points to both collections. Thus, by the Poisson assumption, $E\left(\bar{Y}_{1} \mid N_{R}\right)$ and $E\left(\bar{Y}_{2} \mid N_{g}\right)$ are conditionally (given $N_{g}\left(E_{1}\right), N_{g}\left(E_{2}\right)$ ) independent (being functions of the distinct, independent sets of t-points $t_{1}^{(1)}, t_{2}^{(1)}, \ldots, t_{N_{s}\left(E_{1}\right)}^{(1)}$ and $t_{1}^{(2)}, t_{2}^{(2)}, \ldots, t_{N_{s}\left(E_{2}\right)}^{(2)}$, respectively). Hence,

$$
\begin{aligned}
& E^{N_{k}\left(E_{1}\right) \cdot N_{g}\left(E_{2}\right)}\left\{E\left(\bar{Y}_{1} \mid N_{R}\right) E\left(\bar{Y}_{2} \mid N_{g}\right)\right\} \\
& =E^{N_{g}\left(E_{1}\right) \cdot N_{k}\left(E_{2}\right)}\left[E\left(\bar{Y}_{1} \mid N_{g}\right)\right] E^{\left.N_{R}\left(E_{1}\right) \cdot N_{g}\left(E_{2}\right)\right)}\left\{E\left(\bar{Y}_{2} \mid N_{g}\right)\right\} \\
& =E^{N_{g}\left(E_{1}\right) \cdot N_{g}\left(E_{2}\right)} \bar{Y}_{1} E^{N_{g}\left(E_{1}\right) \cdot N_{g}\left(E_{2}\right)} \bar{Y}_{2}
\end{aligned}
$$


where $E^{N_{k}\left(E_{i}\right), N_{q}\left(E_{2}\right)}\left\{E\left(\bar{Y}_{i} \mid N_{p}\right)\right\}=E^{N_{t}\left(E_{1}\right), N_{q}\left(E_{2}\right)} \bar{Y}_{i}$, since the $\sigma$-algebra generated by the random variables $N_{g}\left(E_{1}\right), N_{g}\left(E_{2}\right)$ is coarser than that generated by the whole process $N_{g}$.

Thus we have shown that

$$
\begin{aligned}
& E^{N_{g}\left(E_{1}\right), N_{s}\left(E_{2}\right)} \bar{Y}_{1} \bar{Y}_{2}-E^{N_{g}\left(E_{t}\right), N_{t}\left(E_{2}\right)} \bar{Y}_{1} E^{\left.N_{k}\left(E_{1}\right) \cdot N_{t}\left(E_{2}\right)\right)} \bar{Y}_{2} \\
& =E^{N_{g}\left(E_{1}\right), N_{g}\left(E_{2}\right)}\left\{E\left(\bar{Y}_{1} \bar{Y}_{2} \mid N_{g}\right)-E\left(\bar{Y}_{1} \mid N_{g}\right) E\left(\bar{Y}_{2} \mid N_{g}\right)\right\}
\end{aligned}
$$

Now note that $E^{N_{f}\left(E_{1}\right), N_{q}\left(E_{2}\right)} \bar{Y}_{i}=E^{N_{f}\left(E_{i}\right)} \bar{Y}_{i}$, and that $E^{N_{x}\left(E_{i}\right)} \bar{Y}_{i}$ is a function of the random variable $N_{g}\left(E_{i}\right)$ only. Also note that because $E_{1}, E_{2}$ are assumed disjoint (since $\rho\left(E_{1}, E_{2}\right)>0$ ), the Poisson process properties imply that the random variables $N_{g}\left(E_{1}\right), N_{g}\left(E_{2}\right)$ are independent. Hence,

$$
\begin{aligned}
& E\left\{E^{N_{\varepsilon}\left(E_{1}\right) \cdot N_{k}\left(E_{2}\right)} \bar{Y}_{1} E^{\left.N_{k}\left(E_{1}\right) \cdot N_{\mathrm{g}}\left(E_{2}\right)\right)} \bar{Y}_{2}\right\}=E\left\{E^{N_{g}\left(E_{1}\right)} \bar{Y}_{1} E^{\left.N_{k}\left(E_{2}\right)\right)} \bar{Y}_{2}\right\} \\
& =E\left\{E^{N_{\varepsilon}\left(N_{1}\right)} \bar{Y}_{1}\right\} E\left(E^{N_{t}\left(E_{2}\right)} \bar{Y}_{2}\right\}=E \bar{Y}_{1} E \bar{Y}_{2}
\end{aligned}
$$

Therefore, taking expectations on both sides of equation (7), we finally arrive at the relation

$$
E \bar{Y}_{1} \bar{Y}_{2}-E \bar{Y}_{1} E \bar{Y}_{2}=E\left(E\left(\bar{Y}_{1} \bar{Y}_{2} \mid N_{R}\right)-E\left(\bar{Y}_{1} \mid N_{R}\right) E\left(\bar{Y}_{2} \mid N_{R}\right)\right)
$$

Conditionally on $N_{g}, \bar{Y}_{i}$ is just a sum of the $N_{g}\left(E_{i}\right)$ random variables $X(\mathrm{t})$ with $t$-indices that happen to be in $E_{i}$. However, for any fixed (conditionally) set of points $\left\{t_{k}, k=1,2, \ldots\right\}$, Minkowski's inequality coupled with the assumed homogeneity of the $X$-process yields

$$
E^{N_{z}}\left|N_{g}\left(E_{i}\right)^{-1} \sum_{k=1}^{N_{z}\left(E_{j}\right)} X\left(t_{k}\right)\right|^{p} \leq E^{N_{k}}|X(\mathrm{t})|^{p}=E|X(\mathrm{t})|^{p}
$$

by the independence of the $X$-process to $N_{g}$. Now, by a well-known mixing incquality (sec Roussas and loannides, 1987), we have that

$$
\left|E\left(\bar{Y}_{1} \bar{Y}_{2} \mid N_{g}\right)-E\left(\bar{Y}_{1} \mid N_{g}\right) E\left(\bar{Y}_{2} \mid N_{g}\right)\right| \leq 10 C_{p}^{2 / p}\left(\alpha_{X}\left(k ;\left|E_{1}\right|,\left|E_{2}\right|\right)\right)^{1-2 / p}
$$

Taking expectations (with respect to $N_{R}$ ) on the above completes the first assertion of the lemma.

To prove the bound concerning the covariance of $\tilde{Y}_{1}$ and $\bar{Y}_{2}$, exactly the same arguments apply only that equation (9) is replaced by

$$
E^{N_{k}}\left|\left(\Lambda_{g}\left(E_{i}\right)\right)^{-1} \sum_{k=1}^{N_{g}\left(E_{i}\right)} X\left(\mathrm{t}_{k}\right)\right|^{p} \leq\left(\frac{N_{g}\left(E_{i}\right)}{\Lambda_{g}\left(E_{i}\right)}\right)^{p} E|X(\mathrm{t})|^{p}
$$

Therefore, using the above bound, we obtain

$$
\begin{aligned}
\mid E\left(\tilde{Y}_{1} \bar{Y}_{2} \mid N_{g}\right) & -E\left(\tilde{Y}_{1} \mid N_{g}\right) E\left(\tilde{Y}_{2} \mid N_{R}\right) \mid \\
& \leq \frac{N_{R}\left(E_{1}\right) N_{g}\left(E_{2}\right)}{\Lambda_{g}\left(E_{1}\right) \Lambda_{g}\left(E_{2}\right)} 10 C_{r}^{2 / p}\left(\alpha_{x}\left(k ;\left|E_{1}\right|,\left|E_{2}\right|\right)\right)^{1-2 / p}
\end{aligned}
$$

Taking expectations (with respect to $N_{g}$ ), and using the independence of $N_{g}\left(E_{1}\right)$ and $N_{g}\left(E_{2}\right)$ and that $E N_{g}\left(E_{i}\right)=\Lambda_{g}\left(E_{i}\right)$ completes the second assertion of the lemma.

Finally, the last two assertions of the lemma involving $\bar{\alpha}$-mixing follow in a similar way to the ones we have proved involving $\alpha$-mixing.

PROOF OF LEMMA 2: Owing to Theorem 1.7.1 of Ivanov and Leonenko (1986) - that actually employs a yet weaker notion than our $\bar{\alpha}_{x}$-mixing, we just need to verify the finiteness of $\int R(t) \mathrm{dt}$ which by necessity will then be equal to the limiting variance of $\frac{1}{\sqrt{|K|}} \int_{K} X(t) d t$; since a covariance kernel is nonnegative definite, the improper integral $\int R(t) d t$ exists, it is nonnegative, but it may be infinite. Note also that, if we are not going to divide by $\int R(t) d t$ to produce a standardized (or uniform) central limit theorem, it is not necessary to prove or assume that $\int R(t) \mathrm{dt} \neq 0$.

Note that by a well-known mixing inequality (sce, for instance, Roussas and loannides, 1987) we have that $|\operatorname{Cov}(X(0), X(t))| \leq$ const. $\bar{\alpha}_{X}\left(\max _{i}\left|t_{i}\right| ; 0\right)^{1-2 /(2+\delta)}$. Thus we have

$$
\begin{aligned}
\int|R(\mathrm{t})| \mathrm{d} t & =O\left(\int_{\left.x_{x}\left(\max _{i}\left|t_{i}\right|: 0\right)^{1-2 /(2+\delta)} \mathrm{dt}\right)}\right. \\
& =O\left(\int_{0}^{\infty} y^{d-1}\left(\frac{1}{y^{\prime+\epsilon}}\right)^{1-2 /(2+\delta)} \mathrm{d} y\right) \\
& =O\left(\int_{0}^{\infty} \frac{1}{y^{1-2 d(2+\delta)^{-1}+\epsilon(1-2 /(2+\delta))}} \mathrm{d} y\right)<\infty
\end{aligned}
$$

where the assumed bound on the $\bar{\alpha}_{X}$-coefficients was used, together with the ; assumption $\epsilon>2 d / \delta$.

PROOF OF THEOREM 2: Note first that $\bar{x}^{\cdot}$ is an average of $/$ i.i.d. random variables, each one being distributed as $\frac{1}{\lambda|| \mid} \int_{B+Y} X(\mathrm{t}) N(\mathrm{dt})$, where $\mathbf{Y}$ has the uniform distribution on $K$.

Therefore,

$$
E^{*} \bar{X}^{*}=E^{*} \frac{1}{\lambda|B|} \int_{B+Y} X^{\prime}(\mathrm{t}) N(\mathrm{dt})=\int_{K} \int_{B+\mathrm{Y}} \frac{1}{\left|K^{\prime}\right| \lambda|B|} X^{\prime}(\mathrm{t}) N(\mathrm{dt}) \mathrm{d} \mathrm{Y}^{\prime}=\bar{X}_{K}
$$


Similarly,

$$
\operatorname{Var}^{*} \bar{X}^{*}=I^{-1} \operatorname{Var}^{*}\left(\frac{1}{\lambda|B|} \int_{B+\mathrm{Y}} X(\mathrm{t}) N(\mathrm{dt})\right)
$$

and

$$
\begin{aligned}
& \operatorname{Var}^{*}\left(\frac{1}{\lambda|B|} \int_{B+Y} X(\mathrm{t}) N(\mathrm{~d} t)\right)=\frac{1}{|K|} \int_{K}\left(\frac{1}{\lambda|B|} \int_{B+Y} X(\mathrm{t}) N(\mathrm{~d} \mathrm{t})-\tilde{X}_{K}\right)^{2} \mathrm{~d} \mathbf{Y} \\
& =\frac{1}{|K|} \int_{K}\left(\frac{1}{\lambda|B|} \int_{B+Y} X(\mathrm{t}) N(\mathrm{dt})-\mu-\left(\bar{X}_{K}-\mu\right)\right)^{2} \mathrm{dY}=A_{1}-A_{2}+A_{3},
\end{aligned}
$$

where

$$
\begin{aligned}
& A_{1}=\frac{1}{|K|} \int_{K}\left(\frac{1}{\lambda|B|} \int_{B+Y} X(\mathrm{t}) N(\mathrm{dt})-\mu\right)^{2} d \mathrm{Y} \\
& A_{2}=\frac{2}{|K|} \int_{K}\left(\frac{1}{\lambda|B|} \int_{B+Y} X(\mathrm{t}) N(\mathrm{dt})-\mu\right)\left(\bar{X}_{K}-\mu\right) \mathrm{d} \mathbf{Y}
\end{aligned}
$$

and

$$
A_{3}=\frac{1}{|K|} \int_{K}\left(\tilde{X}_{K}-\mu\right)^{2} d \mathrm{Y}
$$

Now note that under the assumed conditions, Karr's (1986) theorem implies that $\sqrt{|K|}\left(\bar{X}_{K}-\mu\right) \stackrel{c}{\Longrightarrow} N\left(0, \sigma^{2}\right)$, where $\sigma^{2}=\int R(\mathrm{t}) \mathrm{dt}+R(0) / \lambda$. Observe also that our assumption $R(0)=\operatorname{Var}(X(\mathrm{t}))>0$ implies that $\sigma^{2}>0$, since $\int R(t) \mathrm{dt} \geq 0$ by nonnegative definiteness of $\{R(t)\}$.

Hence, it follows that $\bar{X}_{K}-\mu=O_{P}(1 / \sqrt{|K|})$, and therefore $A_{3}=O_{p}(1 /|K|)$. Similarly, because (also by Karr's (1986) theorem) we have $\frac{1}{\lambda|B|} \int_{n+\gamma} X(\mathrm{t}) N(\mathrm{dt})-\mu=O_{p}(1 / \sqrt{|B|})$, it follows that $A_{2}=O_{P}(1 / \sqrt{|B||K|})$. Since $A_{2}$ and $A_{3}$ are asymptotically negligible, we now focus on $A_{1}$.

Let $G(\mathrm{Y}) \equiv\left(\frac{1}{2 \sqrt{|B|}} \int_{B+\mathrm{Y}} X(\mathrm{t}) N(\mathrm{~d} t)-\mu \sqrt{|B|}\right)^{2}$, and note that due to the homogeneity of the $X$-process and of the point process $N, G(\mathrm{Y})$ is itself a homogeneous random field with index $\mathrm{Y} \in \mathrm{R}^{d}$. Also note that since the $X$ process has more than four finite moments, $E G(\mathrm{Y})^{2}<\infty$, and homogeneity yields $\operatorname{Cov}\left(G\left(\mathrm{Y}_{1}\right), G\left(\mathrm{Y}_{2}\right)\right)=C\left(\mathrm{Y}_{1}-\mathrm{Y}_{2}\right)$ for some covariance function $C(\cdot)$.

So we have

$$
A_{1}=\frac{1}{|K||B|} \int_{K} G(\mathrm{Y}) \mathrm{d} \mathrm{Y}
$$

and

$$
E A_{1}=\frac{1}{|K||B|} \int_{K} E G(\mathrm{Y}) \mathrm{d} \mathrm{Y}=\frac{1}{|B|} E G(0)
$$

by homogeneity of the $G$-field. However, since $|B| \rightarrow \infty$, Karr's (1986) theorem implies that $E G(0) \rightarrow \sigma^{2}$, and thus $E A_{1}=\frac{\sigma^{2}}{|B|}+o(1 /|B|)$.

Now look at

$$
\begin{aligned}
\operatorname{Var}_{1} & =\frac{1}{|K|^{2}|B|^{2}} \int_{K} \int_{K} C\left(\mathrm{Y}_{1}-\mathrm{Y}_{2}\right) \mathrm{dY}_{1} \mathrm{~d} \mathrm{Y}_{2} \\
& =\frac{1}{|K||B|^{2}} \int_{\mathrm{R}^{d}} C(\mathrm{t}) \frac{|K \cap(K-\mathrm{t})|}{|K|} \mathrm{dt}
\end{aligned}
$$

where again the homogeneity of $G$ was used.

Recall that $K$ is the rectangle $\left\{\mathrm{t}=\left(t_{1}, \ldots, t_{d}\right): 0 \leq t_{i} \leq K_{i}, i=1, \ldots, d\right)$ and $B$ is the rectangle $\left\{\mathrm{t}=\left(t_{1}, \ldots, t_{d}\right): 0 \leq t_{i} \leq c K_{i}, i=1, \ldots, d\right\}$. Define "symmetrized" versions of $K$ and $B$ by

$$
K^{*}=\left\{\mathrm{t}=\left(t_{1}, \ldots, t_{d}\right):\left|t_{i}\right| \leq K_{i}, \quad i=1, \ldots, d\right\}
$$

and

$$
B^{*}=\left\{\mathrm{t}=\left(t_{1}, \ldots, t_{d}\right):\left|t_{i}\right| \leq c K_{i}, \quad i=1, \ldots, d\right\}
$$

Now note that

$$
\begin{aligned}
\operatorname{Var} A_{1} & =\frac{1}{|K||B|^{2}} \int_{K^{*}} C(\mathrm{t}) \frac{|K \cap(K-\mathrm{t})|}{|K|} \mathrm{dt} \\
& \leq \frac{1}{|K||B|^{2}} \int_{B^{*}}|C(\mathrm{t})| \mathrm{dt}+\frac{1}{|K||B|^{2}} \int_{\mathrm{K}^{*}-B^{*}}|C(\mathrm{t})| \mathrm{dt} .
\end{aligned}
$$

However, $\frac{1}{\left|K^{\prime}\right||M|^{2}} \int_{n}|C(t)| d t=O\left(\frac{1}{|K||m|}\right)$, since $|C(t)|$ is bounded by $|C(0)|$. Finally note that for $t \in K^{*}-B^{*}$, our Lemma I affords us the possibility of using mixing bounds for the covariance $C(t)$. Letting $p=3$ in Lemma I, we obtain

$$
|C(\mathrm{t})| \leq 10\left(E|G(0)|^{3}\right)^{2 / 3}\left(\bar{\alpha}_{X}\left(\rho\left(\mathrm{t}, B^{*}\right) ;|B|\right)\right)^{1 / 3} ;
$$

but from Lemma 1.8.1 of Ivanov and Leonenko (1986) it follows that $E|G(0)|^{3} \leq$ const. $\left(E|X(t)-\mu|^{6+s}\right)^{6 /(6+s)}$, which is assumed to be finite. Putting all this together, and noting that

$$
|K|^{-1} \int_{K^{*}-B^{*}}\left(\bar{\alpha}_{X}\left(\rho\left(\mathrm{t}, B^{*}\right) ;|B|\right)\right)^{1 / 3} \mathrm{dt} \leq\left|K^{\prime}\right|^{-1} \int_{K^{*}}\left(\bar{\alpha}_{X}(\rho(\mathrm{t}, 0) ;|B|)\right)^{1 / 3} \mathrm{dt}
$$




$$
\begin{aligned}
& \leq|K|^{-1} \int_{0}^{\max K_{i}} y^{j-1}\left(\bar{\alpha}_{x}(y ;|B|)\right)^{1 / 3} \mathrm{~d} y=O\left(\frac{|B|^{\dot{\gamma} / 3}}{|K|} \int_{0}^{\max x_{i} K_{i}} y^{d-1} y^{-\dot{\beta} / 3} \mathrm{~d} y^{\prime}\right) \\
& =O\left(\frac{|B|^{\tilde{\gamma} / 3}}{|K|}\left(\max _{i} K_{i}\right)^{d-\hat{\beta} / 3}\right)=O\left(c^{d \dot{\gamma} / 3}|K|^{\left(\hat{\gamma}-\hat{\beta} d^{-1}\right) / 3}\right)=O(1)
\end{aligned}
$$

becausc $c \rightarrow 0$ and it was assumed that $\bar{\beta} \geq d \bar{\gamma}$. In the above string of inequalitics, the assumed bound on the $\bar{\alpha}_{X}$ cocflicients was used, as well as the fact that $\max _{i} K_{i}=O\left(|K|^{1 / d}\right)$ by assumption. Therefore, it follows that

$$
V_{a r A_{1}}=O\left(\frac{1}{|K||B|}\right)+o\left(\frac{1}{|B|^{2}}\right)=o\left(\frac{1}{|B|^{2}}\right) .
$$

To recapitulatc, we have shown that

$$
\operatorname{Var} \cdot \bar{X}^{*}=E A_{1}+o_{P}\left(\frac{1}{|B|}\right)=\frac{\sigma^{2}}{|B|}+o_{P}\left(\frac{1}{|B|}\right)
$$

Since ||$B|/| K \mid \rightarrow 1$ and $|K| \operatorname{Var} \bar{X}_{K} \rightarrow \sigma^{2}$, it follows that $\frac{\operatorname{Var} \tilde{X}^{2}}{\operatorname{Var} \bar{x}_{K}} \stackrel{P}{\rightarrow} 1$ as claimed.

So far we have shown convergence of the first two bootstrap moments of $\tilde{X} \cdot$ to the corresponding moments of $\bar{X}_{K}$. Since $\bar{X}_{K}$ is asymptotically normal, to complete the proof of Theorem 2 we need to show that $\bar{X}^{*}$ is also asymptotically normal, in $P^{*}$ probability. However, recall that $\tilde{X}^{*}$ is an average of $l$ i.i.d. random variables, with $l \rightarrow \infty$. Therefore, to show that $\bar{X}^{*}$ is asymptotically normal in $P^{*}$ (conditional) probability, it suffices (by the Lyapunov central limit theorem) to show that $E^{*}|G(\mathrm{Y})|^{3 / 2}=O_{p}(1)$. However $E^{*}|G(\mathrm{Y})|^{3 / 2}=\frac{1}{|X|} \int_{K}|G(\mathrm{Y})|^{3 / 2} \mathrm{~d} \mathrm{Y} \equiv \xi$. Due to homogeneity and $\bar{\alpha}$-mixing, a calculation similar to the calculation of $\mathrm{VarA}_{1}$ above yields $\operatorname{Var} \xi \rightarrow 0$. Hence, a weak law of large numbers obtains, and $\xi \stackrel{P}{\longrightarrow} E|G(\mathrm{Y})|^{3 / 2}$ which is finite by Lemma 1.8 .1 of Ivanov and Leonenko (1986) and the assumed finiteness of $6+\delta$ moments of the $X$-process. Therefore, $E^{*}|G(Y)|^{3 / 2}=O_{P}(1)$ and the proof is completed.

PROOF OF THEOREM 3: Note again that $E^{*} \bar{X}^{*}$ is an average of $/$ i.i.d. random variables, each one being distributed as $\frac{1}{N(B+\gamma)} \int_{n+\gamma} X(\mathrm{t}) N(\mathrm{dt})$, where $\mathrm{Y}$ has the uniform distribution on $K$.
Therefore,

$$
\begin{aligned}
E \bar{X}^{*} & =E^{*} \frac{1}{N\left(B+\mathrm{Y}^{\prime}\right)} \int_{B+Y} X(\mathrm{t}) N(\mathrm{dt}) \\
& =\int_{K} \int_{B+\mathrm{Y}} \frac{1}{|K| N(B+\mathrm{Y})} X(\mathrm{t}) N(\mathrm{dt}) \mathrm{d} \mathrm{Y}=\bar{X}_{K}
\end{aligned}
$$

by a conditioning (on $N$ ) argument similar to the proof of the unbiasedness of $\bar{X}_{R}$ as an estimator of $\mu$-see Karr $(1986,1991)$.

Firthermore, recill that under the assumed conditions, Karr's (1986) theorem implies that $\sqrt{|K|}\left(\bar{X}_{K}-\mu\right) \stackrel{\mathcal{c}}{\Longrightarrow} N\left(0, \sigma^{2}\right)$, where $\sigma^{2}=\int R(t) d t+R(0) / \lambda$.

Now due to the Poisson assumption, we have $N(K) /|K| \rightarrow \lambda$ almost surely. As a matter of fact, $E N(K)=\operatorname{Var} N(K)=\lambda|K|$; therefore, $\frac{N(K)}{\lambda|K|}=1+O_{P}\left(\frac{1}{\sqrt{|K|}}\right)$. By the aforegoing discussion, we have that

$$
\begin{aligned}
\sqrt{|K|}\left(\tilde{X}_{K}-\bar{X}_{K}\right) & =\sqrt{|K|}\left(\tilde{X}_{K^{\prime}}-\mu+\mu-\tilde{X}_{K}\right) \\
& =\frac{N(K)-\lambda|K|}{N(K) \lambda \sqrt{|K|}} \int_{K}(X(\mathrm{t})-\mu) N(\mathrm{dt}) \\
& =O_{P}\left(N(K)^{-1} \int_{K}(X(\mathrm{t})-\mu) N(\mathrm{dt})\right)=O_{P}\left(\frac{1}{\sqrt{|K|}}\right)=o_{P}(1)
\end{aligned}
$$

since $\bar{X}_{K}-\dot{\mu}=O_{P}\left(\frac{1}{\sqrt{|K|}}\right)$ by Karr's (1986) result.

Consequently, $\sqrt{|K|}\left(\tilde{X}_{K}-\mu\right)$ and $\sqrt{|K|}\left(\bar{X}_{K}-\mu\right)$ have the same asymptotic distribution, as well as the same asymptotic variance (by Karr's (1986) theorem); thus Theorem 3 follows from Theorem 2.

PROOF OF THEOREM 4: The proof is similar to the proof of Theorem 2; below we point out only the differences and new elements.

Note again that $\tilde{X}^{*}$ is an average of $/$ i.i.d. random variables, each one being distributed as $\frac{1}{\lambda|B|} \int_{B+\mathrm{Y}} X(\mathrm{t}) N(\mathrm{dt})$, where $\mathrm{Y}$ has the uniform distribution on $K_{1-c}$.

Therefore,

$$
E^{*} \bar{X}^{*}=E^{*} \frac{1}{\lambda|B|} \int_{B+Y} X(\mathrm{t}) N(\mathrm{dt})=\int_{K_{1-\gamma}} \int_{n_{+Y}} \frac{1}{\lambda|B| \mid K_{1-c \mid}} X(\mathrm{t}) N(\mathrm{~d} t) d Y
$$

Recall that

$$
E^{*} \tilde{X}^{*}=\int_{K} \int_{B+\gamma} \frac{1}{\lambda|B|\left|K^{*}\right|} X(\mathrm{t}) N(\mathrm{~d} t) \mathrm{d} \mathrm{Y}=\bar{X}_{K}
$$


Hence,

$$
\begin{aligned}
|K|\left(\tilde{X}_{K}-\mu\right)-\left|K_{1-d}\right|\left(E^{*} \bar{X}^{*}-\mu\right) & =\int_{K-K_{1-\varepsilon}} \int_{B+Y} \frac{1}{\lambda|B|}(X(\mathrm{t})-\mu) N(\mathrm{dt}) \mathrm{d} \mathrm{Y} \\
& =\int_{K-K_{1-\epsilon}} O_{P}(1 / \sqrt{|B|}) d \mathrm{Y} \\
& =O_{P}\left(\left|K-K_{1-c}\right| / \sqrt{|B|}\right)
\end{aligned}
$$

However $\left|K_{1-c}\right|=(1-c)^{d}|K|$, and $\left|K-K_{1-c}\right|=O(c|K|)$. Therefore,

$$
\begin{aligned}
E^{*} \bar{X}^{*} & =\frac{\tilde{X}_{K}-\mu}{(1-c)^{d}}+\mu+O_{P}(c / \sqrt{|B|})=\mu+O_{P}(1 / \sqrt{|K|})+O_{P}(c / \sqrt{|B|}) \\
& =\mu+o_{P}(1 / \sqrt{|B|})
\end{aligned}
$$

Similarly,

$$
\begin{aligned}
\mid V_{a r^{*}} \tilde{X}^{*} & =\frac{1}{\left|K_{1-c}\right|} \int_{K_{1-r}}\left(\frac{\mathrm{l}}{\lambda|B|} \int_{B+\mathrm{Y}} X(\mathrm{t}) N(\mathrm{~d} \mathrm{t})-E^{*} \bar{X}^{*}\right)^{2} \mathrm{~d} \mathrm{Y} \\
& =\frac{1}{\left|K_{1-c}\right|} \int_{K_{1-r}}\left(\frac{1}{\lambda|B|} \int_{B+\mathrm{Y}} X(\mathrm{t}) N(\mathrm{~d} \mathrm{t})-\mu-\left(E^{*} \tilde{X}^{*}-\mu\right)\right)^{2} \mathrm{~d} \mathrm{Y} \\
& =D_{1}-D_{2}+D_{3},
\end{aligned}
$$

where

$$
\begin{aligned}
& D_{1}=\frac{1}{\left|K_{1-c}\right|} \int_{K_{1-r}}\left(\frac{1}{\lambda|B|} \int_{B+\mathrm{Y}} X(\mathrm{t}) N(\mathrm{dt})-\mu\right)^{2} \mathrm{~d} \mathrm{Y} \\
& D_{2}=\frac{2}{\left|K_{1-c}\right|} \int_{K_{1-\varepsilon}}\left(\frac{1}{\lambda|B|} \int_{B+\mathrm{Y}} X(\mathrm{t}) N(\mathrm{dt})-\mu\right)\left(E^{*} \bar{X}^{*}-\mu\right) \mathrm{d} \mathrm{Y}
\end{aligned}
$$

and

$$
D_{3}=\frac{1}{\left|K_{1-c}\right|} \int_{K_{1-e}}\left(E^{*} \bar{X}^{*}-\mu\right)^{2} \mathrm{~d} \mathrm{Y}
$$

However, necessarily $D_{3}=o_{P}(1 /|B|)$, since we have shown that $E^{*} \tilde{X}^{*}$ $=\mu+o_{P}(1 / \sqrt{|B|})$. Similarly, $D_{2}=o_{P}(1 /|B|)$, since $\frac{1}{\lambda|B|} \int_{B+\gamma} X(\mathrm{t}) N(\mathrm{dt})-\mu$ $=O_{P}(1 / \sqrt{|B|})$.

Note now that $D_{1}$ is of the same form as the quantity $A_{1}$ in the proof of Theorem 2; the only differences are: (a) integrating/averaging takes place over $K_{1-c}$ as opposed to $K$, (b) $K$ is not a rectangle anymore, but rather a general compact, convex set, and (c) we generally define "symmetrized" versions of the nonrectangular $K_{1-c}$ and $B$ by $K_{i-c}^{*}=$ convex hull of $\left\{t:|t| \in K_{1-c}\right\}$, and $B^{*}=$ convex hull of $\{t:|t| \in B\}$, where $|t| \equiv\left(\left|t_{1}\right|, \ldots,\left|t_{d}\right|\right)$. However, the same arguments used in the proof of Theorem 2 to show that $A_{1}=\frac{\sigma^{2}}{|B|}+o_{P}(1 /|B|)$ can be used to show $|B| D_{1} \stackrel{P}{\rightarrow} \sigma^{2}$, which in turns implies that $\frac{\operatorname{Var}^{\prime} \tilde{X}^{*}}{\operatorname{Var}_{x}} \stackrel{P}{\longrightarrow} 1$. Finally, the proof of asymptotic normality follows verbatim the proof of Theorem 2 .

PROOF OF THEOREM S: The proof follows from Theorem 4 using the same arguments used in the proof of Theorem 3.

\section{REFERENCES}

Bickel, P. and Freedman, D. (1981), Some asymptotic theory for the bootstrap, Ann. Stat., 9, 1196-1217.

Carlstein, E. (1986), The use of subseries values for estimating the variance of a general statistic from a stationary sequence, Ann. Stat., 14, 11711179.

Cressie, N. (1991), Statistics for Spatial Data, New York: Wilcy.

Daley, D.J. and Vera-Jones, D. (1988), An Introduction to the Theory of Point Processes, New York: Springer.

Doukhan, P. (1994), Mixing: Properties and Examples, Lecture Notes in Statistics No. 85, New York: Springer.

Efron, B. and Tibshirani, R. (1993), An Introduction to the Bootstrap, New York: Chapman \& Hall.

Götzc, F. and Künsch, H.R. (1996), Second order correctness of the blockwise bootstrap for stationary observations, Ann. Stat., 24, 1914-1933.

Hall, P. (1985), Resampling a coverage pattern, Stochastic Process. Appl.. 20, 231-246.

Hall, P. (1992), The Bootstrap and Edgeworth Expansion, New York: Springer.

Hall, P., Horowitz, J.L., and Jing, B. (1996), On blocking rules for the block bootstrap with dependent data, Biometrika, 82, 561-574.

Ivanov, A.V. and Leonenko, N.N. (1986), Statistical Analysis of Random Fields, Dordrecht: Kluwer.

Karr, A. F. (1986), Inference for stationary random fields given Poisson samples, Adv. Appl. Prob., 18, 406-422. 
Karr, A. F. (1991), Point Processes and their Statistical Inference, 2nd edn., New York: Marcel Dekker.

Krickeberg, K. (1982), Processus ponctucls en statistique, École d' été de probabilités de Saint-Flour X-1980, In: P.L. Hennequin (ed.), Lecture Notes in Mathematics No, 929, Berlin: Springer, pp. 205-313.

Künsch, H.R. (1989), The jackknife and the bootstrap for general stationary observations, Anm. Stat., 17, 1217-1241.

Lahiri, S.N. (1991), Sccond order optimality of stationary bootstrap, Stat. Prob. Lett., 11, 335-341.

Liu, R.Y. and Singh, K. (1992), Moving blocks jackknife and bootstrap capture weak dependence, In: R. LePage and L. Billard (eds.), Exploring the Limits of Bootstrap, Wiley, New York, pp. 225-248.

Masry, E. (1988), Random sampling of continuous-parameter stationary processes: Statistical properties of joint density estimators, J. Multivar. Anal., 36, 133-165.

Politis, D.N. and Romano, J.P. (1992a), A general resampling scheme for triangular arrays of $\alpha$-mixing random variables with application to the problem of spectral density estimation, Ann. Stat., 20, 1985-2007.

Politis, D.N. and Romano, J.P. (1992b), A nonparametric resampling procedure for multivariate confidence regions in time series analysis, In C. Page and R. LePage (eds.), Computing Science and Statistics, Proceedings of the 22nd Symposium on the Interface, Springer, New York, pp. 98-103.

Politis, D.N. and Romano, J.P. (1992c), A circular block-resampling procedure for stationary data, In: R. LePage and L. Billard (eds.), Exploring the Limits of Bootstrap, Wiley, New York, pp. 263-270.

Politis, D.N. and Romano, J.P. (1993). Nonparametric resampling for homogeneous strong mixing random fields, J. Multivar. Anal., 47, 301328.

Politis, D.N. and Romano, J.P. (1994). The stationary bootstrap, J. Am. Stat. Assoc., 89, 1303-1313.

Raïs, N. (1992). Méthodes de reéchantillonage et de sous échantillonage dans le contexte spatial et pour des données dépendantes, PhD Thesis, Department of Mathematics and Statistics, University of Montreal, Montreal.

Ripley, B.D. (1981), Spatial Statistics, Wiley, New York.
Rosenblatt, M. (1985), Stationary Sequences and Random Fields, Boston, MA: Birkhäuser.

Roussas, G.G. and Ioannides, D. (1987), Moment inequalities for mixing sequences of random variables, Stochastic Anal. Appl., 5, 61-120.

Shao, J. and Tu, D. (1995), The Jackknife and Bootstrap, New York: Springer.

Sherman, M. and Carlstein, E. (1994), Nonparametric estimation of the moments of a general statistic computed from spatial data, J. Am. Stat. Assoc., 89, 496-500.

Sherman, M. and Caristein, E. (1996), Replicate histograms, J. Am. Siat. Assoc., 91, 566-576.

Yadrenko, M.I. (1983), Spectral Theory of Random Fields, New York: Optimization Software. 